\title{
A Probabilistic Power Estimation Method for Combinational Circuits Under Real Gate Delay Model*
}

\author{
G. THEODORIDIS ${ }^{a, \dagger}$, S. THEOHARIS ${ }^{a}$, D. SOUDRIS ${ }^{b, \ddagger}$ \\ and C. GOUTIS ${ }^{\mathrm{a}}$ \\ ${ }^{a}$ VLSI Design Lab., Dept. of Electrical and Computer Eng., University of Patras, 26110, Greece; \\ ${ }^{\mathrm{b}}$ VLSI Design and Testing Center, Dept. of Electrical and Computer Eng., Democritus University of Thrace, \\ 67100 Xanthi, Greece
}

(Received 24 September 1999; In final form 6 October 1999)

\begin{abstract}
Our aim is the development of a novel probabilistic method to estimate the power consumption of a combinational circuit under real gate delay model handling temporal, structural and input pattern dependencies. The chosen gate delay model allows handling both the functional and spurious transitions. It is proved that the switching activity evaluation problem assuming real gate delay model is reduced to the zero delay switching activity evaluation problem at specific time instances. A modified Boolean function, which describes the logic behavior of a signal at any time instance, including time parameter is introduced. Moreover, a mathematical model based on Markov stochastic processes, which describes the temporal and spatial correlation in terms of the associated zero delay based parameters is presented. Based on the mathematical model and considering the modified Boolean function, a new algorithm to evaluate the switching activity at specific time instances using Ordering Binary Decision Diagrams (OBBDs) is also presented. Comparative study of benchmark circuits demonstrates the accuracy and efficiency of the proposed method.
\end{abstract}

Keywords: Low power design; Switching activity estimation; Power dissipation model; Markov chains; Temporal and spatial correlation; CMOS combinational circuits

\section{INTRODUCTION}

Power dissipation is recognized as a critical parameter in modern VLSI design field. The development of competitive market sectors such as wireless applications, laptops, and portable medical devices, depend on the power consumption as the most important parameter, because the

* "Portions reprinted, with permission, from Proceedings of 1999 IEEE International Symposium on Circuits and Systems (ISCAS), May 30-June 2, 1999, Orlando, Florida, USA, pp. Vol. I 286-289, (C) 1999-IEEE".

†e-mail: theodor@ee.upatras.gr

${ }^{\ddagger}$ Corresponding author. e-mail: dsoudris@,demokritos.cc.duth.gr 
growth rate of the battery technologies is not so promising. In addition, problems such as chip overheating, electomigration and hot electrons are strongly-depended on power dissipation [1,2]. For these reasons low power design techniques at all levels of the design flow ranging from the layout level up to architectural and system level have been xdeveloped $[1,2]$. However, an estimation of the power consumption at any design level, is required. Thus simultaneously with low power design techniques a large number of power estimation methods have been also developed $[2,3]$.

The power dissipation at gate level is proportional to the switching activity of the circuit nodes, because in the current technologies the dynamic power dissipation is by far the most dominated factor comparing with the power consumption coming from the short circuit and leakage current. According to their mathematical model, the power estimation methods of the combinational circuits are categorized as probabilistic methods and statistical ones. Moreover, considering the assumed gate delay model they are also characterized as zero- and real-gate delay methods. A survey of the power estimation methods for combinational logic circuits has been reported in $[2,3]$.

Assuming zero-gate delay model $[4,5]$ and real gate delay model [6-9], a number of probabilistic power estimation methods have been presented. In particular, a method for calculating the switching activity of the circuit nodes, using Order Binary Decision Diagrams (OBBDs) was proposed [4]. Modeling the behavior of the logic signal as an one step Markovian process the first order temporal correlation was captured. Moreover, structural correlation, which is coming from the reconvergent fanout nodes, was also considered by partitioning the circuit using techniques from the chip testing field. However, the input signals were assumed to be mutually-independent, making this method inaccurate for highly correlated input streams. In [5], a probabilistic method, which captures all the types of correlations was presented. Specifically, modeling the logic signal as a Markovian process, the temporal correlation was considered, while the input pattern dependency was also captured by the concepts of the spatiotemporal transition correlation coefficient and the signal isotropy. Using OBDDs, the structural correlation was considered by propagating the estimated switching activities and spatiotemporal coefficients through the circuit nodes. In [6], the concept of the probability waveforms to estimate the average power dissipation was presented. The probability waveforms consist of the signal probability and the signal transition probability value. Given the probability waveforms of the primary inputs, an algorithm to estimate the probability waveforms of the internal nodes of the circuit has been introduced. However, both the structural and input dependencies were not taken into consideration. An extension of the probability waveforms is the tagged probabilistic simulation approach presented in [7]. In particularly, at each node the transition probability is broken in four cases: stable to 0 , stable to 1 , perform a low to high transition $(0 \rightarrow 1)$ and a high to low $(1 \rightarrow 0)$ transition. Inertial delays, hazards, temporal correlation of the primary inputs and structural correlations were considered by this method. However, the primary inputs were assumed spatially uncorellated, while simultaneous transitions were not considered. In [8], a symbolic simulation algorithm has been proposed using OBDDs. The structural and the first-order temporal correlation were handled but the input pattern dependency was not considered. A new method for calculating the transition probabilities by performing symbolic polynomial simulation was proposed in [9]. It is based on the signal probability evaluation method of [11], which has been extended to handle temporal correlation and arbitrary transport gate delay models. The method was parameterized by a single factor, which determines the circuit levels over which the structural correlation is considered. A propagation algorithm of the estimated switching activities through the circuit nodes has also been presented. However, the input signals were also considered mutually-independent making this method inaccurate. 
In this paper a novel probabilistic method for accurate power estimation of a combinatorial circuit is introduced. Taking into account the temporal, structural and input pattern dependency of the circuit signals and considering simultaneous transitions, the switching activity of any logic circuit node is calculated under a real delay gate model. Since the proposed method includes all the types of data correlations, it can be considered as an extension of the approach of [5]. Although the [5] can capture all the types of correlations, is valid only for zero delay model. In contrast, the proposed method derives accurate results because it captures all the possible signal transitions, i.e., the functional transition and the spurious transitions (or glitches).

It is proved that under a proper formulation, the switching activity estimation problem assuming real gate delay model can be transformed to a zero delay switching activity estimation problem at specific time instances. For this reason a new Boolean function including time parameter and a proper mathematical model are established. The concepts of transition probability and spatiotemporal correlation coefficient, which are derived from the zero delay switching activity estimation field, are extended to estimate the switching activity under real delay model. Also, it is proved that the transition probabilities and transition correlation coefficients of the primary inputs at any time interval can be evaluated by the zero delay transition probabilities and transition correlation coefficients. Moreover, a method for the switching activity evaluation of circuit nodes in different time instances using OBDDs is also introduced. Employing a set of benchmark circuits, comparisons with a switch level simulation and the method of [9] prove the efficiency of the proposed method.

The paper is organized, as follows: in Section 2 the problem formulation is presented, the mathematical model is introduced in Section 3, while the switching activity evaluation algorithm is given in Section 4. The experimental results are presented in Section 5 while the conclusions and future work are discussed in Section 6.

\section{PROBLEM FORMULATION}

The power estimation problem of a combinational logic circuit, under real gate delay model can be stated as:

\begin{abstract}
"Given the gate level description of a combinational circuit with $N$ inputs and $M$ outputs and the inertial delays of its gates, and, assuming that the period of the applied input vectors is greater or equal to the settling time of the circuit, estimate the average power consumption of the circuit for an input vector stream through the calculation of the circuit average switching activity."
\end{abstract}

The accuracy of the switching activity evaluation is strongly depended on the data correlation of the circuit signals and the assumed gate delay model. Concerning data correlation, it includes the temporal and spatial correlation. By temporal correlation we mean the dependency of a signal on its previous values. The spatial correlation is divided to the structural correlation, which is coming from the reconvergent fanout nodes, and the input pattern dependency coming from the sequence of the applied input vectors. In zero delay model, a gate perform at most one transition in a clock cycle, which is called functional or useful transition. However, under real delay model the gate may perform additional transitions called spurious transitions or glitches. Therefore, all types of signals correlations under real delay model have to be considered to estimate the power dissipation accurately.

The output of a gate generates a glitch, if two conditions are satisfied: (i) the necessary condition, which requires the difference of the transition arrival times of the input signals to be equal or greater than the inertial delay of the gate and (ii) the sufficient condition, which requires the appropriate transitions of the input signal(s) to switch the gate output. Consequently, the time parameter plays an important role in the glitch generation. For that purpose, a modified Boolean function including time parameter, which will describe the exact 
behavior of a logic signal in time domain, is needed. Manipulating this modified logic function and considering the probabilistic properties of its variables the switching activity can be calculated accurately.

Example We assume a logic circuit with gate delay equals to one delay unit, $d$, as it shown in Figure 1. The logic behavior of the node $f$ can be described in time domain as follows:

$$
f=F\left(x_{1}, x_{2}, t\right)=x_{1}(t-2 d) x_{2}(t-2 d) x_{2}(t-d)
$$

The signal $f$ may switch in two time instances, i.e., $t_{1}^{f}=d$ and $t_{2}^{f}=2 d$. More specifically, the transition of the signal $f$ at $t_{1}^{f}=d$ depends on the transitions of the primary inputs $x_{1}$ and $x_{2}$ at time points $t_{1}^{x_{1}}=-d, t_{1}^{x_{1}}=-d$, and $t_{1}^{x_{2}}=0$, while the transition of $f$ at $t_{2}^{f}=2 d$ depends on the transitions of the signals $x_{1}$ and $x_{2}$ at $t_{2}^{x_{1}}=0$, $t_{2}^{x_{2}}=0$, and $t_{2}^{x_{2}}=d$. The corresponding logic functions of $f$, which are derived by (1), of time instances $t_{1}$ and $t_{2}$ are: $f_{1}=F\left(x_{1}, x_{2}, d\right)=$ $x_{1}(-d) x_{2}(-d) x_{2}(0)$ and $f_{2}=F\left(x_{1}, x_{2}, 2 d\right)=x_{1}(0)$ $x_{2}(0) x_{2}(d)$, respectively.

From the above example, we infer that the time parameter plays critical role in the generation of the switching activity. Additionally, the logic behavior of signal $f$ is described entirely by the modified logic function of Eq. (1) at any time instance. The general form of this function is given bellow:

$$
\begin{aligned}
f\left(t, x_{1}, x_{2}, \ldots, x_{n}\right) & =f\left(\tilde{x}\left(t-k_{1}\right), \ldots, \tilde{x}_{p}\left(t-k_{p}\right)\right) \\
\text { with } \quad k_{i} & =\sum_{j} d_{j}
\end{aligned}
$$

where, $k_{i}$ is the delay of the path $\pi_{i}$ and $d_{j}$ is the delay of the $j$ th gate, $p$ is the number of paths, $\tilde{x}_{k}=x_{i}$ if $\pi_{k}$ starts at input $x_{i}$. This function describes the logic behavior of a signal in time domain, while in the specific time instances it is reduced to an ordinary Boolean function, where the Boolean variables are the corresponding logic values of the input signals of these time points.

Having as starting point Eq. (2), a novel mathematical model, which describes the behavior of a logic signal in terms of time should be introduced. We aim at the development of a new method, which reduces the power estimation problem considering real delay model to a zero delay problem at certain switching time points. For that purpose, we introduce new concepts and formulas, which express parameters of real delay modeled power estimation problem in terms of zero delay parameters. Specifically, we provide the suitable material for temporal correlation modelling, extending the concept of transition probability for certain time intervals. Then, it follows a set of formally-proven new formulas of the generalised transition correlation coefficients, which describe the spatiotemporal correlation among different signals.

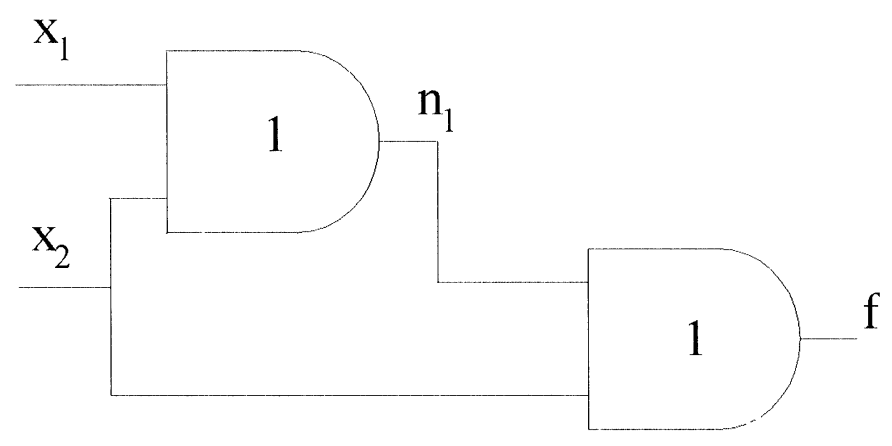

FIGURE 1 The logic circuit with Unit Delay AND gates. 


\section{MATHEMATICAL MODEL}

The behavior of a binary signal, $x$, at a time point, $t$, i.e., $x(t)$, is modelled as a random variable of a time homogeneous, Strict Sense Stationary, lagone Markov stochastic process having two states, $s$, with $s \in S=\{0,1\}[10]$.

The transition probability, $p_{k l}^{x}(t)$, expresses the probability of a signal $x$ to perform a transition from the state $k$ to the state $l$ within two successive time points $t-1$ and $t$. That is:

$p_{k l}^{x}(t)=p(x(t-1)=k \wedge x(t)=l) \quad \forall k, l \in S$

The switching activity, $E(x, t)$, of a signal $x$ at time instance $t$ is given by:

$$
E(x, t)=p_{01}^{x}(t)+p_{10}^{x}(t)
$$

where $p_{01}^{x}(t)$ (or $p_{10}^{x}(t)$ ) is the transition probability of the signal $x$ to perform the transition from state 0 to 1 (or 1 to 0 ).

The above stochastic process models the behavior of an input signal at times $t=0, t=T, t=2 T$, etc., where the input signal performs a transition. However, as it can be seen in Eq. (1), the transition probabilities $p_{k l}^{x}(t)$ of an input signal $x$ at multiple time points $t= \pm a d(a=1,2)$ (i.e., $p_{k l}^{x_{1}}(0), p_{k l}^{x_{1}}(-d)$ and $\left.p_{k l}^{x_{1}}(d)\right)$, are needed.

Definition 1 A Signal Transition Probability Vector, $\boldsymbol{P}^{x}(t)$, of a signal $x$ at a time instance $t$, is defined as the vector of all transition probabilities $p_{k l}^{x}(t)$, with $k, l \in S$ :

$$
\boldsymbol{P}^{x}(t)=\left(p_{00}^{x}(t), p_{01}^{x}(t), p_{10}^{x}(t), p_{11}^{x}(t)\right)
$$

We introduce the transition probability concept of an input signal in time intervals $(-T, 0)$ and $(0$, $T)$ as $p_{k k}^{x}\left(0^{-}\right)$and $p_{l l}^{x}\left(0^{+}\right)$, respectively. It should be noticed that within these time intervals the input signal does not perform transition according to the problem formulation. Their corresponding values are computed by the next lemma.

LEMMA 1 The transition probability of an input signal, $x$, at a time point $t \in\left\{0,0^{+}, 0^{-}\right\}$is expressed in terms of the transition probability at $t=0$ as follows:

$$
\boldsymbol{P}^{x}(t)=f\left(\boldsymbol{P}^{x}(0)\right)
$$

and are computed by:

$$
\begin{gathered}
p_{k k}^{x}\left(0^{-}\right)=p_{k k}^{x}(0)+p_{k(1-k)}^{x}(0) \quad \forall k \in S \\
p_{l l}^{x}\left(0^{+}\right)=p_{l l}^{x}(0)+p_{(1-l) l}^{x}(0) \quad \forall l \in S \\
p_{k l}^{x}\left(0^{-}\right)=p_{k l}^{x}\left(0^{+}\right)=0 \quad \forall k, l \in S \wedge k \neq l
\end{gathered}
$$

Proof The proof of the above lemma is given in the Appendix I.

The above lag-one stochastic process and the one step transition probabilities (Eq. (3)) ensure that the first-order temporal correlation of a signal $x$ can be described entirely. However, the accurate the power estimation implies that the spatial correlation among the circuit signals should be considered. Since we use a real delay gate model, the concept of Transition Correlation Coefficient, $T C$, [5] should be generalised for capturing the spatiotemporal correlation of two signals for any two certain time instances. The $T C$ for a zerodelay model is [5]:

$$
\begin{aligned}
& T C_{k l, m n}^{x_{1}, x_{2}} \\
& \quad=\frac{p\left(x_{1}(t-1)=k \wedge x_{1}(t)=l \wedge x_{2}(t-1)=m \wedge x_{2}(t)=n\right)}{p\left(x_{1}(t-1)=k \wedge x_{1}(t)=l\right) p\left(x_{2}(t-1)=m \wedge x_{2}(t)=n\right.}
\end{aligned}
$$

The dependency among three signals $x_{1}, x_{2}$ and $x_{3}$, is expressed by the pairwise coefficients of (6) as follows:

$$
\begin{aligned}
T C_{k l o, m n p}^{x_{1}, x_{2}, x_{3}}= & T C_{k l, m n}^{x_{1}, x_{2}} T C_{k o, m p}^{x_{1}, x_{3}} T C_{l o, n p}^{x_{2}, x_{3}} \\
& \text { with } \quad k, l, m, n, o, p \in\{0,1\}
\end{aligned}
$$

Definition 2 A Generalised Transition Correlation Coefficient, $T C_{k l, m n}^{x_{1}, x_{2}}\left(t_{1}, t_{2}\right)$, between the signals $x_{1}$ and $x_{2}$, which perform transitions from the states $k$ to $l$ and from $m$ to $n$, at times $t_{1}$ and $t_{2}$, 
respectively, is defined as:

$$
T C_{k l, m n}^{x_{1}, x_{2}}\left(t_{1}, t_{2}\right)=\frac{p\left(x_{1}\left(t_{1}-1\right)=k \wedge x_{1}\left(t_{1}\right)=l \wedge x_{2}\left(t_{2}-1\right)=m \wedge x_{2}\left(t_{2}\right)=n\right)}{p\left(x_{1}\left(t_{1}-1\right)=k \wedge x_{1}\left(t_{1}\right)=l\right) p\left(x_{2}\left(t_{2}-1\right)=m \wedge x_{2}\left(t_{2}\right)=n\right)}
$$

where $k, l, m, n \in S$.

Since the proposed method is a "global" one, we have to introduce appropriate generalized TCs between any two input signals, taking into account their spatiotemporal dependency. For that purpose, three time points for any input signal, i.e., $t=0^{+}, t=0, t=0^{-}$(where $0^{+} / 0^{-}$denotes the time intervals $(-T, 0) /(0, T))$, are needed.

Definition 3 The Transition Probability Coefficient Vector, $\boldsymbol{T} \boldsymbol{C}^{x_{1}, x_{2}}\left(t_{1}, t_{2}\right)$, between two signals $x_{1}$ and $x_{2}$ at time instances $t_{1}$ and $t_{2}$ is defined as:

$$
\begin{aligned}
& \boldsymbol{T C}^{x_{1}, x_{2}}\left(t_{1}, t_{2}\right)=\left(T C_{00,00}^{x_{1}, x_{2}}\left(t_{1}, t_{2}\right),\right. \\
& \left.T C_{00,01}^{x_{1}, x_{2}}\left(t_{1}, t_{2}\right), \ldots, T C_{11,11}^{x_{1}, x_{2}}\left(t_{1}, t_{2}\right)\right)
\end{aligned}
$$

According to Eq. (9), the transition probability coefficients vector at $t_{1}=0$ and $t_{2}=0$ contains the sixteen TCs of Eq. (7). That is:

$$
\boldsymbol{T C}^{x_{1}, x_{2}}(0,0)=\left(T C_{00,00}^{x_{1}, x_{2}}, \ldots, T C_{11,11}^{x_{1}, x_{2}}\right)
$$

Lemma 2 The spatiotemporal correlation coefficients of two input signals $x_{1}$ and $x_{2}$, at time points $t_{1}, t_{2} \in\left\{0,0^{+}, 0^{-}\right\}$are expressed by:

$$
\boldsymbol{T} \boldsymbol{C}^{x_{1}, x_{2}}\left(t_{1}, t_{2}\right)=f\left(\boldsymbol{T} \boldsymbol{C}^{x_{1}, x_{2}}(0,0), \boldsymbol{P}^{x_{1}}(0), \boldsymbol{P}^{x_{2}}(0)\right)
$$

and can be calculated by:

$$
\begin{aligned}
& T C_{k k, m n}^{x_{1}, x_{2}}\left(0^{-}, 0\right)=\frac{T C_{k k, m n}^{x_{1}, x_{2}}(0,0) p_{k k}^{x_{1}}(0)+T C_{k(1-k), m n}^{x_{1}, x_{2}}(0,0) p_{k(1-k)}^{x_{1}}(0)}{\left(p_{k k}^{x_{1}}(0)+p_{k(1-k)}^{x_{1}}(0)\right) p_{m n}^{x_{2}}(0)} \\
& T C_{l l, m n}^{x_{1}, x_{2}}\left(0^{+}, 0\right)=\frac{T C_{l l, m n}^{x_{1}, x_{2}}(0,0) p_{l l}^{x_{1}}(0)+T C_{(1-l) l, m n}^{x_{1}, x_{2}}(0,0) p_{(1-l) l}^{x_{1}}(0)}{\left(p_{l l}^{x_{1}}(0)+p_{(1-l) l}^{x_{1}}(0)\right) p_{m n}^{x_{2}}(0)} \\
& T C_{k k, m m}^{x_{1}, x_{2}}\left(0^{-}, 0^{-}\right)=\frac{T C_{k k, m m}^{x_{1}, x_{2}}(0,0) p_{k k}^{x_{1}}(0) p_{m m}^{x_{2}}(0)+T C_{k k, m(1-m)}^{x_{1}, x_{2}}(0,0) p_{k k}^{x_{1}}(0) p_{m(1-m)}^{x_{2}}(0)}{p_{k k}^{x_{1}}(0) p_{m m}^{x_{2}}(0)+p_{k k}^{x_{1}}(0) p_{m(1-m)}^{x_{2}}(0)+p_{k(1-k)}^{x_{1}}(0) p_{m m}^{x_{2}}(0)+p_{k(1-k)}^{x_{1}}(0) p_{m(1-m)}^{x_{2}}(0)}+ \\
& +\frac{T C_{k(1-k), m m}^{x_{1}, x_{2}}(0,0) p_{k(1-k)}^{x_{1}}(0) p_{m m}^{x_{2}}(0)+T C_{k(1-k), m(1-m)}^{x_{1}, x_{2}}(0,0) p_{k(1-k)}^{x_{1}}(0) p_{m(1-m)}^{x_{2}}(0)}{p_{k k}^{x_{1}}(0) p_{m m}^{x_{2}}(0)+p_{k k}^{x_{1}}(0) p_{m(1-m)}^{x_{2}}(0)+p_{k(1-k)}^{x_{1}}(0) p_{m m}^{x_{2}}(0)+p_{k(1-k)}^{x_{1}}(0) p_{m(1-m)}^{x_{2}}(0)} \\
& T C_{l l, n n}^{x_{1}, x_{2}}\left(0^{+}, 0^{+}\right)=\frac{T C_{l l, n n}^{x_{1}, x_{2}}(0,0) p_{l l}^{x_{1}}(0) p_{n n}^{x_{2}}(0)+T C_{l l,(1-n) n}^{x_{1}, x_{2}}(0,0) p_{l l}^{x_{1}}(0) p_{(1-n) n}^{x_{2}}(0)}{p_{l l}^{x_{1}}(0) p_{n n}^{x_{2}}(0)+p_{l l}^{x_{1}}(0) p_{(1-n) n}^{x_{2}}(0)+p_{(1-l) l}^{x_{1}}(0) p_{n n}^{x_{2}}(0)+p_{(1-l) l}^{x_{1}}(0) p_{(1-n) n}^{x_{2}}(0)}+ \\
& +\frac{T C_{(1-l) l, n n}^{x_{1}, x_{2}}(0,0) p_{(1-l) l}^{x_{1}}(0) p_{n n}^{x_{2}}(0)+T C_{(1-l) l,(1-n) n}^{x_{1}, x_{2}}(0,0) p_{(1-l) l}^{x_{1}}(0) p_{(1-n) n}^{x_{2}}(0)}{p_{l l}^{x_{1}}(0) p_{n n}^{x_{2}}(0)+p_{l l}^{x_{1}}(0) p_{(1-n) n}^{x_{2}}(0)+p_{(1-l) l}^{x_{1}}(0) p_{n n}^{x_{2}}(0)+p_{(1-l) l}^{x_{1}}(0) p_{(1-n) n}^{x_{2}}(0)}
\end{aligned}
$$

where $k, l, m, n \in S$. 
Proof The proof of the above lemma is given in the Appendix I.

\section{SWITCHING ACTIVITY EVALUATION ALGORITHM}

Definition 4 We define as Valid Time Points Vector, $\boldsymbol{T}^{x}=\left(t_{1}, \ldots, t_{r}\right)$, all the possible transition time points for a signal $x$.

These time points are derived by performing a traversal of the circuit from the primary inputs to primary outputs considering the delay of the gates. Consequently, the switching activity estimation problem is reduced to the estimation of $\boldsymbol{P}^{x}(t)$ $\forall t \in \boldsymbol{T}^{x}$. The calculation procedure of switching activity (i.e., estimation of $\left.p_{k l}^{x}\left(t_{i}\right)\right)$ is similar to the zero-delay method to [5] and consists of the following steps:

(a) Construct the OBDD for any time point $t_{i} \in \boldsymbol{T}^{x}$,

(b) Find the sets of all paths of the OBDD that results into the leaf node $k, \Pi_{k}^{x}$,

(c) Find the sets of all paths of the OBDD that results into the leaf node $l, \Pi_{l}^{x}$ and

(d) Combine any path of $\Pi_{k}^{x}$, with any path of the set $\Pi_{l}^{x}$ :

$$
p_{k l}^{x}=\sum_{\pi \in \Pi_{k}^{x}} \sum_{\pi^{\prime} \in \Pi_{k}^{x}} \prod_{i=1}^{v}\left(p_{k_{i} l_{i}}^{x_{i}} \prod_{1 \leq i<j \leq v} T C_{k_{i} l_{i}, k_{j} l_{j}}^{x_{i}, x_{j}}\right)
$$

\section{EXPERIMENTAL RESULTS}

The proposed power estimation method is implemented by ANSI C language, while its efficiency is proved by a number of ISCAS' 85 benchmark multilevel circuits. For technology mapping, a library of primitive gates up to 5 primary inputs, is used. All power estimations are measured in $\mu \mathrm{W}$ with $20 \mathrm{MHz}$ clock frequency and $5 \mathrm{~V}$ power supply. Also, a gate capacitance is assumed to be $C_{g}=0.05 \mathrm{pF}$, while a node with a fan-out $F$ has capacitance $F^{*} C_{g}$. For comparison reasons, three categories of input vectors: (i) without spatial correlation (column NO), (ii) with low spatial correlation (column LOW) and (iii) with high spatial correlation (column HIGH), are chosen. For each category and circuit and for reliability reasons, 15 input vector files of 20000 vectors are generated. These input vector files are used both for estimation and simulation.

For a signal $x$, we define as switching activity error the quantity $\operatorname{Err}(x)=\left|E_{\text {eff }}(x)-E_{\text {eff }}^{\prime}(x)\right| / E_{\text {eff }}$ $(x)$, where $E_{\text {eff }}(x)$ is the real switched capacitance of signal $x$ and $E_{e f f(x)}^{\prime}$ is the estimated one. For a combinational circuit with $L$ signals and a specific input vector set $V_{j}$, we define as Total Power Consumption the quantity Power $\left(V_{j}\right)=(1 / 2) V_{d d}^{2} \cdot f \cdot \sum_{i-1}^{L}$ $E_{\text {eff }}\left(x_{i}\right)$, as Total Error the quantity Total Error $=$ $\left|\operatorname{Power}\left(V_{j}\right)-\operatorname{Power}\left(V_{j}\right)^{\prime}\right| \operatorname{Power}\left(V_{j}\right)$, as Mean Error the quantity Mean Error $\left(V_{j}\right)=\sum_{i=1}^{L} \operatorname{Err}\left(x_{i}\right) / N$ and finally as Maximum Error the quantity Max Error $\left(V_{j}\right)=\max \left\{\operatorname{Err}\left(x_{1}\right), \operatorname{Err}\left(x_{2}\right), \ldots, \operatorname{Err}\left(x_{L}\right)\right\}$. Choosing $K$ input vector sets the above formulas become as shown in the following: Power ${ }^{K}=\sum_{j=1}^{K}$ $\operatorname{Power}\left(V_{j}\right)$, Total Error ${ }^{K}=\sum_{j=1}^{K} \operatorname{Power}\left(V_{j}\right)-\sum_{j=1}^{K}$ $\operatorname{Power}\left(V_{j}\right)^{\prime} \quad \sum_{j=1}^{K} \operatorname{Power}\left(V_{j}\right)$, which is denoted as TOTAL $^{K}$, MeanError ${ }^{K}=\sum_{j=1}^{K}$ Mean Error $/ K$, which is denoted as $\operatorname{MEAN}^{K}$, and MaxError ${ }^{K}=$ $\max \left\{\right.$ MeanError $\left(V_{1}\right), \ldots$, Mean Error $\left.\left(V_{K}\right)\right\}$, which is denoted as $\mathrm{MAX}^{K}$. In our experimental procedure $K=15$.

We compare the proposed method and the method [9], which to best of our knowledge is the most accurate real delay power estimation method, with Mentor's Graphics QUICKSIM II simulator. The power consumption differences between each method and switch level simulator are depicted in Tables I and II.

Table I gives the error in power estimation (\%) of the proposed method. The average TOTAL ${ }^{K}$ error is about $0.02 \%$ for $\mathrm{NO}$ spatial input correlation, $1.4 \%$ for LOW spatial correlation, and $1.5 \%$ for HIGH spatial correlation. The corresponding average $\mathrm{MEAN}^{K}$ error values are $0.6 \%, 3.3 \%$ and $3.6 \%$, while the average MAX $^{K}$ 
TABLE I Real power estimation errors of the prop. method

\begin{tabular}{|c|c|c|c|c|c|c|c|c|c|}
\hline \multirow[b]{2}{*}{ Circuit } & \multicolumn{3}{|c|}{ Total $^{K}$} & \multicolumn{3}{|c|}{$\operatorname{Mean}^{K}$} & \multicolumn{3}{|c|}{$\operatorname{Max}^{K}$} \\
\hline & No & Low & High & No & Low & High & No & Low & High \\
\hline c17 & 0,051 & 0,263 & 0,630 & 0,238 & 1,040 & 1,580 & 0,519 & 1,704 & 2,729 \\
\hline $\mathrm{Cm} 163$ & 0,018 & 0,915 & 0,394 & 0,704 & 3,192 & 3,249 & 1,298 & 4,307 & 4,463 \\
\hline $\mathrm{Cm} 42$ & 0,024 & 0,351 & 0,124 & 0,953 & 2,768 & 1,902 & 1,741 & 4,142 & 3,117 \\
\hline $\mathrm{Cm} 82$ & 0,027 & 3,600 & 3,603 & 0,223 & 5,192 & 6,009 & 0,391 & 10,186 & 11,833 \\
\hline $\mathrm{cu}$ & 0,010 & 0,017 & 0,425 & 0,501 & 1,131 & 1,623 & 0,649 & 1,581 & 2,076 \\
\hline decod & 0,010 & 0,635 & 0,065 & 1,472 & 4,137 & 3,274 & 3,665 & 7,009 & 5,266 \\
\hline Majority & 0,017 & 0,765 & 2,133 & 0,349 & 3,224 & 4,910 & 1,004 & 4,531 & 6,125 \\
\hline $\mathrm{pml}$ & 0,002 & 1,128 & 0,866 & 1,191 & 4,193 & 4,336 & 2,182 & 5,983 & 6,913 \\
\hline rca4 & 0,023 & 4,871 & 5,274 & 0,283 & 5,368 & 6,072 & 0,705 & 8,893 & 9,901 \\
\hline Average & 0,020 & 1,394 & 1,502 & 0,657 & 3,361 & 3,662 & 1,350 & 5,371 & 5,825 \\
\hline
\end{tabular}

TABLE II Real power estimation errors of method [9]

\begin{tabular}{|c|c|c|c|c|c|c|c|c|c|}
\hline \multirow[b]{2}{*}{ Circuit } & \multicolumn{3}{|c|}{$\operatorname{Max}^{K}$} & \multicolumn{3}{|c|}{$\operatorname{Mean}^{K}$} & \multicolumn{3}{|c|}{$\operatorname{Max}^{K}$} \\
\hline & No & Low & High & No & Low & High & No & Low & High \\
\hline $\mathrm{c} 17$ & 0,082 & 6,202 & 8,324 & 0,794 & 11,145 & 14,965 & 2,682 & 17,375 & 23,364 \\
\hline $\mathrm{Cm} 163$ & 0,007 & 5,564 & 7,560 & 1,179 & 14,534 & 19,934 & 3,067 & 21,379 & 29,597 \\
\hline $\mathrm{Cm} 42$ & 0,027 & 1,802 & 2,465 & 1,259 & 15,756 & 21,685 & 2,139 & 21,857 & 30,253 \\
\hline $\mathrm{Cm} 82$ & 0,018 & 23,117 & 31,10 & 0,550 & 32,19 & 43,232 & 0,965 & 76,959 & 103,31 \\
\hline $\mathrm{cu}$ & 0,009 & 2,473 & 3,378 & 0,814 & 6,105 & 8,162 & 1,335 & 7,647 & 10,277 \\
\hline decod & 0,020 & 7,264 & 9,920 & 2,162 & 20,535 & 28,694 & 5,952 & 33,511 & 47,048 \\
\hline Majority & 0,032 & 4,638 & 6,289 & 0,687 & 12,851 & 17,288 & 1,312 & 19,682 & 26,461 \\
\hline $\mathrm{pm} 1$ & 0,009 & 5,321 & 7,446 & 1,465 & 18,893 & 26,889 & 2,650 & 29,298 & 42,269 \\
\hline rca4 & 0,014 & 20,666 & 28,296 & 0,735 & 25,114 & 34,465 & 1,492 & 37,947 & 52,379 \\
\hline Average & 0,024 & 8,561 & 11,642 & 1,072 & 17,458 & 23,924 & 2,399 & 29,517 & 40,552 \\
\hline
\end{tabular}

errors are $1.3 \%, 5.3 \%$ and $5.8 \%$. The input stream with NO spatial is derived by a pseudo random generator, the LOW spatial is derived by an LFSR and the HIGH spatial by the output of a counter.

The increased errors for LOW and HIGH spatial correlation input streams coming from the fact that we consider only the first order transition probabilities and the transition correlation coefficient is a pairwise coefficient. However, as it is shown in Table $\mathrm{I}$, the maximum error is about $5 \%$, while only in one case it exceeds $10 \%$. Hence, the modeling of the switching behavior of a signal by the first order transition probabilities and the modeling of the signals correlation by the first order pairwise correlation coefficients are adequate for a gate level power estimation. In similar manner, we may consider higher order dependencies but the complexity of the problem is increased.
Table II shows the power estimation errors of [9] for the same input vectors and benchmarks. It can be seen that for NO spatial correlation, the error values are small, whereas the average errors of LOW and HIGH spatial correlation are large, i.e., $8.5 \%$ and $11.6 \%$ for TOTAL $^{K}$ power, $17.5 \%$ and $23.8 \%$ for MEAN $^{K}$ power, and up to $30 \%$ and $40.7 \%$ for $\mathrm{MAX}^{K}$ power, respectively. The increased errors coming from the fact that the input pattern dependencies are not considered, because the input signals are assumed mutually independent.

Employing the proposed method and [9], the associated total power consumption of benchmarks circuits is shown in Table III. It can seen that the lack of spatial correlations in the primary inputs increases the power estimation error (e.g., for HIGH correlation, the MAX error of circuit 
TABLE III Total power dissipation in $\mu \mathrm{W}$

\begin{tabular}{|c|c|c|c|c|c|c|}
\hline \multirow[b]{2}{*}{ Circuit } & \multicolumn{3}{|c|}{ Proposed } & \multicolumn{3}{|c|}{ [9] } \\
\hline & No & Low & High & No & Low & High \\
\hline $\mathrm{c} 17$ & 935,000 & 937,938 & 929,588 & 934,950 & 993,350 & 1014,425 \\
\hline $\mathrm{cm} 163$ & 3310,488 & 3341,375 & 3324,125 & 3309,425 & 3495,113 & 3558,588 \\
\hline $\mathrm{cm} 42$ & 3444,600 & 3455,863 & 3448,050 & 3444,563 & 3505,338 & 3528,025 \\
\hline $\mathrm{cm} 82$ & 2023,088 & 2096,475 & 2096,538 & 2022,600 & 2493,463 & 2650,875 \\
\hline $\mathrm{cu}$ & 12427,16 & 12430,51 & 12375,65 & 12409,75 & 12713,38 & 12822,60 \\
\hline decod & 3415,038 & 3437,063 & 3413,150 & 3416,838 & 3665,025 & 3750,688 \\
\hline maj. & 1048,438 & 1040,225 & 1025,888 & 1048,400 & 1097,625 & 1114,100 \\
\hline pm1 & 4183,313 & 4230,450 & 4219,463 & 4185,338 & 4406,163 & 4492,813 \\
\hline rca4 & 4024,413 & 4221,438 & 4237,625 & 4024,425 & 4856,800 & 5167,45 \\
\hline
\end{tabular}

$\mathrm{CU}$ is $103 \%$ ) making the method of [9] inefficient in terms of accuracy for correlated input streams.

\section{CONCLUSIONS}

Assuming a real gate delay model, we have proposed a novel method for the estimation of the power dissipation of a logic circuit. The method constitutes an extension of the zero delay probabilistic method [5] and takes into account the first-order temporal and the spatial correlations. A modified Boolean function, which describes the logic behavior of a signal in time domain, and extension of the basic concepts of the zero delay power estimation models have been introduced. The accuracy of the method has been proved, while the importance of the input pattern dependencies in terms of accuracy has been shown in the analysis of the experimental results.

Since the proposed method is a global approach, our future work is to implement a method that propagates the primary input statistics and correlation coefficients through the logic network. Moreover, the proposed method could be extended to capture the pulse filtering and elimination capacitance in a chain of gates by considering a range of inertial gate delays $\left[d_{\min }, d_{\max }\right]$ and taking into account the load capacitance. In addition, the basic concepts and the proposed method could be modified to take place in the power estimation of the sequential circuits.

\section{Acknowledgements}

This work was partially supported by LPGD project ESPRIT IV 25256 of European Union.

\section{References}

[1] Rabaey, J. and Pedram, M., "Low Power Design Methodologies", Kluwer Academic Publishers, 1996.

[2] Nebel, W. and Mermet, J., "Low Power Design in Deep Submicron Electronics", Kluwer Academic Publishers, 1997.

[3] Najm, F., "A Survey of Power Estimation Techniques in VLSI circuits", In: IEEE Trans. on VLSI, 2(4), 446-455, December, 1995.

[4] Schneider, P. and Schlichmann, U., "Decomposition of Boolean functions for low power based on a new power estimation technique", In: Proc. of Int. Workshop on Low Power Design, pp. 123-128, Napa Valley, CA, April, 1994.

[5] Marculescu, R., Marculescu, D. and Pedram, M. (1995). "Efficient Power estimation for highly correlated input streams", In: Proc. of Design Automation Conference (DAC), pp. 628-634.

[6] Najm, F., Burch, R., Yang, P. and Haij, I., "Probabilistic Simulation for Reliability Analysis of CMOS VLSI circuits", In: IEEE Trans. on CAD, 9(4), 439 - 450, Apr., 1990.

[7] Tsui, C.-Y., Pedram, M., Chen, C.-A. and Despain, A. M., "Efficient Estimation of Dynamic Power Dissipation Under a Real Delay Model", In: Proc. of IEEE Int. Conference on CAD, pp. 224-228, November, 1993.

[8] Monteiro, J., Ghosh, A., Devadas, S., Keutzer, K. and White, J., "Estimation of average switching activity in combinatorial and sequential circuits", In: IEEE Trans. on CAD, 16(1), 121 - 127, January, 1997.

[9] Costa, J. C., Monteiro, J. C. and Devadas, S., "Switching Activity Estimation using Limited Depth Reconvergent Path analysis", In: Proc. of Int. Symp. On Low Power Electronics and Design (ISLPED), pp. 184 - 189, Monterey CA, August 18-20, 1997.

[10] Papoulis, A., "Probabilities, Random Variables and Stochastic Processes", Mc. Graw-Hill Co., 1984.

[11] Parker, K. P. and McCluskey, J., "Probabilistic Treatment of General Combinational Networks", IEEE Trans. on Computers, C24, 668-670, June, 1975. 


\section{APPENDIX I}

Proof of Lemma 1 The stochastic process of an input signal at time point $t$ has as one-step probability matrix the matrix $Q(t)$ where:

$$
Q(t)=\left[\begin{array}{ll}
\pi_{00}^{x}(t) & \pi_{01}^{x}(t) \\
\pi_{10}^{x}(t) & \pi_{11}^{x}(t)
\end{array}\right]
$$

with $0 \leq \pi_{k l}^{x}(t) \leq 1, \quad \sum_{l} \pi_{k l}^{x}(t)=1 \quad$ and $k, l \in S$, where each entry, $\pi_{k l}^{x}(t)$, is the one-step conditional probability, which can be defined by:

$$
\pi_{k l}^{x}(t)=\frac{p_{k l}^{x}(t)}{p_{k}^{x}(t)} \quad \forall k, l \in S
$$

It is assumed that the stochastic process is time homogeneous and therefore, ChapmanKolmogorov equations are taken place [12]. Thus, we obtain that:

$$
\boldsymbol{P}(t)=\boldsymbol{P}(t) Q(t)
$$

where, $\boldsymbol{P}(t)=\left[p_{0}^{x}(t), p_{1}^{x}(t)\right]$ are the steady state probability vector at time $t$. The stochastic processes at $t=0$ and $t=0^{-}$is shown in the following
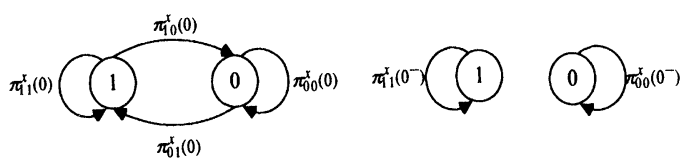

(a)

(b)

Markov Chains of input signal $x$ : (a) $t=0$, (b) $t=0^{-}$.

The corresponding one-step probability matrices $Q(0)$ and the $Q\left(0^{-}\right)$are:

$$
\begin{gathered}
Q(0)=\left[\begin{array}{ll}
\pi_{00}^{x}(0) & \pi_{01}^{x}(0) \\
\pi_{10}^{x}(0) & \pi_{11}^{x}(0)
\end{array}\right] \\
Q\left(0^{-}\right)=\left[\begin{array}{cc}
\pi_{00}^{x}\left(0^{-}\right) & 0 \\
0 & \pi_{11}^{x}\left(0^{-}\right)
\end{array}\right]
\end{gathered}
$$

Using Eqs. (I.4) and (I.5) and solving Eq. (I.3), it is obtained that:

$$
\boldsymbol{P}(0)=\boldsymbol{P}(0) Q(0)
$$

$$
\begin{aligned}
& p_{0}^{x}(0)= p_{1}^{x}(0) \pi_{10}^{x}(0)+p_{0}^{x}(0) \pi_{00}^{x}(0) \\
& p_{1}^{x}(0)= p_{1}^{x}(0) \pi_{11}^{x}(0)+p_{0}^{x}(0) \pi_{01}^{x}(0) \\
& \boldsymbol{P}\left(0^{-}\right)=\boldsymbol{P}\left(0^{-}\right) Q\left(0^{-}\right) \\
& p_{1}^{x}\left(0^{-}\right)=p_{1}^{x}\left(0^{-}\right) \pi_{11}^{x}\left(0^{-}\right) \\
& p_{0}^{x}\left(0^{-}\right)=p_{0}^{x}\left(0^{-}\right) \pi_{00}^{x}\left(0^{-}\right)
\end{aligned}
$$

Additionally, the stochastic process is SSS and thus,

$$
\begin{gathered}
p_{1}^{x}\left(0^{-}\right)=p_{1}^{x}(0)=p_{1}^{x}\left(0^{+}\right)=p_{1}^{x} \\
p_{0}^{x}\left(0^{-}\right)=p_{0}^{x}(0)=p_{0}^{x}\left(0^{+}\right)=p_{0}^{x} \\
p_{0}^{x}+p_{1}^{x}=1
\end{gathered}
$$

Then, from Eqs. (I.7), (I.8) (I.10) (I.11), (I.12), (I.13) and (I.14), it is concluded that:

$$
\begin{aligned}
& \pi_{11}^{x}\left(0^{-}\right)=\pi_{11}^{x}(0)+\pi_{10}^{x}(0) \\
& \pi_{00}^{x}\left(0^{-}\right)=\pi_{00}^{x}(0)+\pi_{01}^{x}(0)
\end{aligned}
$$

Eventually, multiplying the associated left-hand and right-hand side terms of Eqs. (I.15) and (I.16) with $p_{k}^{x}(0)$, it is obtained:

$$
\begin{gathered}
p_{k k}^{x}\left(0^{-}\right)=p_{k k}^{x}(0)+p_{k(1-k)}^{x}(0) \quad \forall k \in S \\
p_{k l}^{x}\left(0^{-}\right)=0 \quad \forall k, l \in S \wedge k \neq l
\end{gathered}
$$

Working in similar way, we can prove for $t=0^{+}$.

Proof of Lemma 2 We prove the formula of Eq. (11), $T C_{l l, m n}^{x_{1}, x_{2}}\left(0^{-}, 0\right)$. The remaining formulas, i.e., Eqs. (11.1), (11.2), (11.3), (11.4) and (11.5) can be proved similarly. By definition [5], the $T C$ for the zero delay model is:

$$
T C_{k l, m n}^{x_{1}, x_{2}}(0,0)=\frac{p\left(x_{1}^{k l}(0) \wedge x_{2}^{m n}(0)\right)}{p\left(x_{1}^{k l}(0)\right) p\left(x_{2}^{m n}(0)\right)}
$$

where $k$ and $l$ are the logic values before a transition and $m$ and $n$ the values after a transition of 
the signals $x_{1}$ and $x_{2}$, respectively and $k, l, m$, $n \in S$. From Definition 2, $T C_{k k, m n}^{x_{1}, x_{2}}\left(0^{-}, 0\right)$ can be expressed as:

$$
T C_{k k, m n}^{x_{1}, x_{2}}\left(0^{-}, 0\right)=\frac{p\left(x_{1}^{k k}\left(0^{-}\right) \wedge x_{2}^{m n}(0)\right)}{p\left(x_{1}^{k k}\left(0^{-}\right)\right) p\left(x_{2}^{m n}(0)\right)}
$$

Applying Lemma 1 (i.e., Eq. (4)) and substituting the event $x_{1}^{k k}\left(0^{-}\right)$in terms of $t=0$ into (I.20), we obtain:

$$
\begin{aligned}
& T C_{k k, m n}^{x_{1}, x_{2}}\left(0^{-}, 0\right) \\
& \quad=\frac{p\left(\left(x_{1}^{k k}(0) \vee x_{1}^{k(1-k)}(0)\right) \wedge x_{2}^{m n}(0)\right)}{\left(p\left(x_{1}^{k(1-k)}(0)\right)+p\left(x_{1}^{k k}(0)\right) p\left(x_{2}^{m n}(0)\right)\right.}
\end{aligned}
$$

Since the events $x_{1}^{k k}(0)$ and $x_{1}^{k(1-k)}(0)$ are mutually disjoint, it is obtained that:

$$
\begin{aligned}
& T C_{k k, m n}^{x_{1}, x_{2}}\left(0^{-}, 0\right) \\
& \quad=\frac{p\left(x_{1}^{k k}(0) \wedge x_{2}^{m n}(0)\right)+p\left(x_{1}^{k(1-k)}(0) \wedge x_{2}^{m n}(0)\right)}{\left(p\left(x_{1}^{k(1-k)}(0)\right)+p\left(x_{1}^{k k}(0)\right)\right) p\left(x_{2}^{m n}(0)\right)}
\end{aligned}
$$

By definition of $T C$ [5] at $t=0$, and using Eq. (I.19) it holds:

$$
\begin{aligned}
p\left(x_{1}^{k l}(0)\right. & \left.\wedge x_{2}^{m n}(0)\right) \\
= & p\left(x_{1}^{k l}(0)\right) p\left(x_{2}^{m n}(0)\right) T C_{k l, m n}^{x_{1}, x_{2}}(0,0)
\end{aligned}
$$

where $k, l, m, n \in S$.

Eventually, from Eqs. (I.22) and (I.23) we infer that:

$$
\begin{aligned}
& T C_{k k, m n}^{x_{1}, x_{2}}\left(0^{-}, 0\right) \\
& \quad=\frac{T C_{k k, m n}^{x_{1}, x_{2}}(0,0) p_{k k}^{x_{1}}(0)+T C_{k(1-k), m n}^{x_{1}, x_{2}}(0,0) p_{k(1-k)}^{x_{1}}(0)}{p_{k k}^{x_{1}}(0) p_{m n}^{x_{2}}(0)+p_{k(1-k)}^{x_{1}}(0) p_{m n(0)}^{x_{2}}}
\end{aligned}
$$

\section{Authors' Biographies}

George Theodoridis received his Diploma in Electrical Engineering from the University of Patras, Greece, in 1994. Since then, he is currently working towards to Ph.D. at Electrical Engineering,
University of Patras. His research interests include low power design, logic synthesis, computer arithmetic, and power estimation.

Spyros Theoharis received his Diploma in Computer Engineering and Informatics from the University of Patras, Greece, in 1994. Since then, he is currently working towards to Ph.D. at Electrical Engineering, University of Patras. His research interests include low power design, multilevel logic synthesis, parallel architectures, and power estimation.

Dr. Dimitrios Soudris received his Diploma in Electrical Engineering from the University of Patras, Greece, in 1987. He received the Ph.D. Degree from in Electrical Engineering, from the University of Patras in 1992. He is currently working as Ass. Professor in Dept. of Electrical and Computer Engineering, Democritus University of Thrace, Greece. His research interests include parallel architectures, computer arithmetic, vlsi signal processing, and low power design. He has published more than 30 papers in international journals and conferences. $\mathrm{He}$ is a member of the IEEE and ACM.

Dr. Costas Goutis was a Lecturer at School of Physics and Mathematics at the University of Athens, Greece, from 1970 to 1972. In 1973, he was Technical Manager in the Greek P.P.T. He was Research Assistant and Research fellow in the Department of Electrical Engineering at the University of Strathclyde. U.K., from 1976 to 1979, and Lecturer in the Department of Electrical and Electronic Engineering at the University of Newcastle upon Tyne, U.K., from 1979 to 1985. Since he has been Associate Professor and Full Professor in the Department of Electrical and Computer Engineering, University of Patras, Greece. His recent interests focus on VLSI Circuit Design, Low Power VLSI Design, Systems Design, Analysis and Design of Systems for Signal Processing and Telecommunications. $\mathrm{He}$ has published more than 110 papers in international journals and conferences. He has been awarded a large number of Research Contracts from ESPRIT, RACE, and National Programs. 

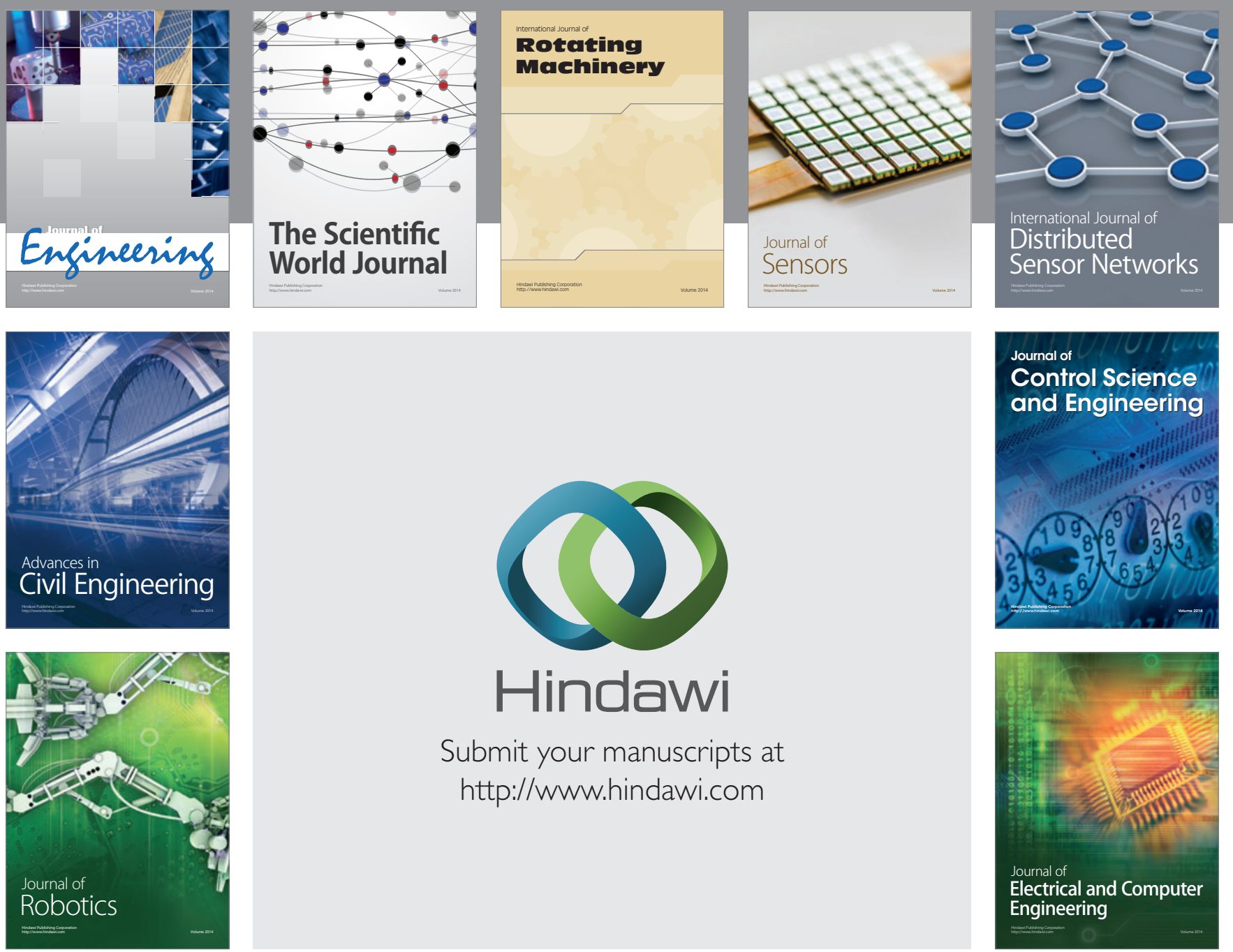

Submit your manuscripts at

http://www.hindawi.com
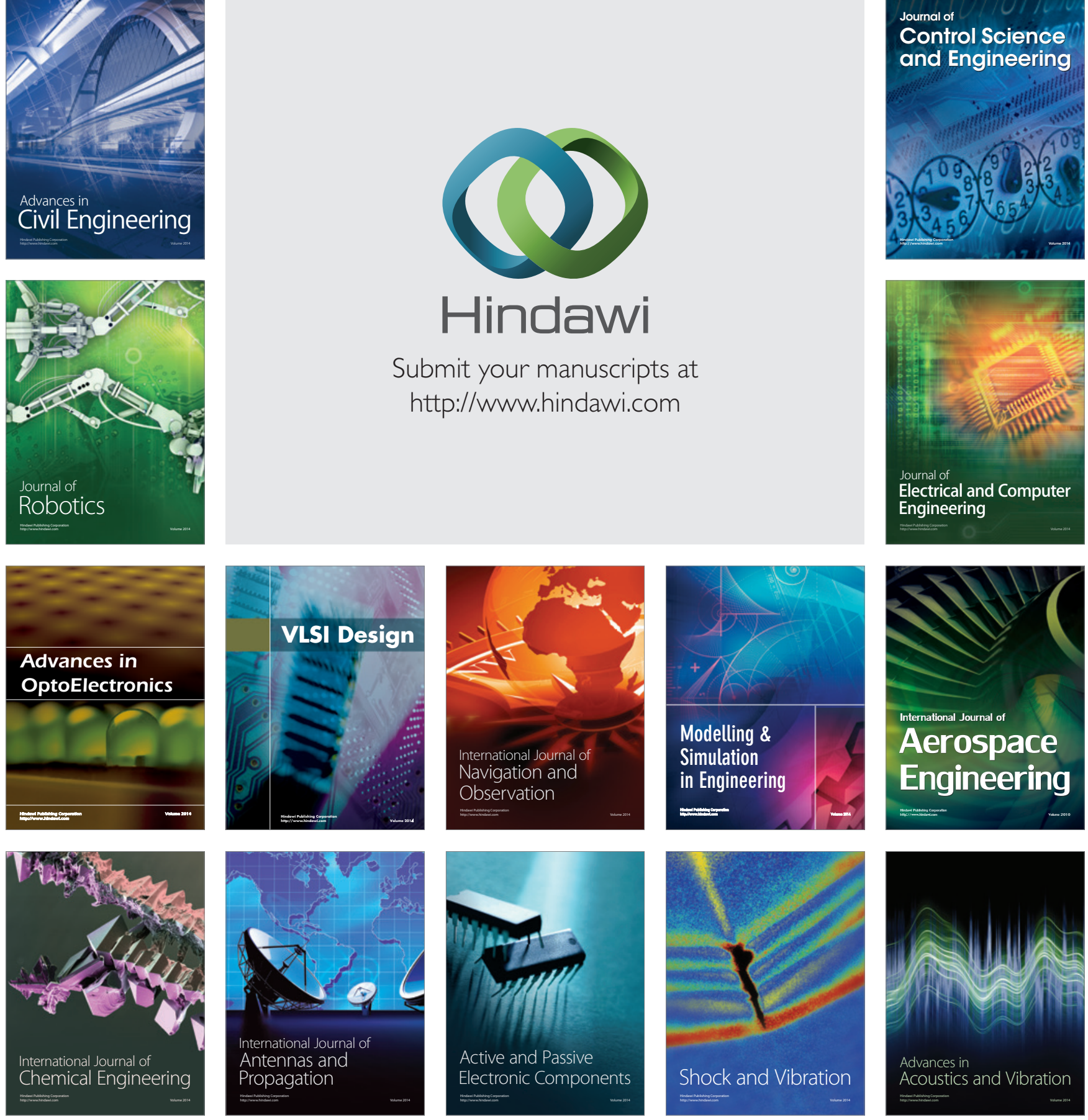